\title{
Amélioration de la mesure et de la prévision de l'ingestibilité des fourrages chez le mouton par la prise en compte des variations de la capacité d'ingestion à l'aide d'un fourrage témoin
}

\author{
Jean Pierre Dulphy, René Baumont, Louis L'Hotelier, \\ Camille Demarquilly \\ Avec la collaboration technique de Marinette Jailler, Jacqueline Jamot \\ et Aline Détour
}

\author{
Équipe Valeur Alimentaire, Station de Recherches sur la Nutrition des Herbivores \\ Inra, Theix, 63122, Saint-Genès-Champanelle, France
}

\begin{abstract}
Improvement of the measurement and prediction of forage ingestibility in sheep by taking into account the variations of their intake capacity using a standard forage. Sheep present important variations of their intake capacity that are related, for example, to the photoperiod. This makes it difficult to estimate the ingestibility of conserved forages, because they may be fed at different periods of the year. A method is proposed to standardise the measure of this forage ingestibility and to make it independent of the main factors influencing the intake capacity. Thus, the ingestibility of 51 forages was measured on 1- to 2-year-old Texel wethers. To correct the effect of different factors ( the photoperiod and the group effect) which influence the intake capacity of these animals, a standard hay was fed before and after all sequences of measurements. The evolution of intake capacity was estimated with this hay, for each group of six sheep, for each period of measurement, by linear interpolation between two measures concerning the standard hay. Under these conditions, it appears that: 1) the intake capacity of wethers leads to an under-estimation of forage ingestibility in December-January and to a overestimation in May-September; 2) the proposed correction is well justified; indeed, the relationship between ingestibility and NDF content, a classical prediction criteria, gives a residual standard deviation of $9.9 \mathrm{~g} \mathrm{DM} \cdot \mathrm{kg}^{-1}$ light weight $(\mathrm{LW})^{0.75}$ before correction, and to 5.2 after correction; 3 ) the correction makes it possible to conclude that ingestibility of well-cured hays and silages is nearly equivalent to initial fresh forages. It is proposed to use this method of correction each time, when measurements of ingestibility are achieved on sheep, at different seasons, and/or with different group of animals, and when the results have to be pooled. (@ Elsevier/Inra)
\end{abstract}

ingestibiliy / forages / ingestive capacity / sheep / methodology

Résumé - Les ovins présentent des variations importantes de leur capacité d'ingestion liées, entre autres facteurs, à la photopériode. Ceci rend difficile l'estimation de l'ingestibilité des fourrages conservés, car leur mesure peut avoir lieu à différentes périodes de l'année. Cette note propose une

Tél. : 04736240 77; fax : 0473624273 ; dulphy@ clermont.inra.fir 
méthodologie qui permet de mesurer l'ingestibilité des fourrages indépendamment des facteurs de variation liés à la capacité d'ingestion des moutons. L'ingestibilité de 51 fourrages a été mesurée avec des béliers castrés Texel, âgés de 1 à 2 ans. Pour corriger l'effet de variations dans les capacités d'ingestion de ces animaux un foin standard a été distribué avant et après toutes les séquences de mesures. À partir des quantités ingérées de ce foin, l'évolution de la capacité d'ingestion a été estimée, lot par lot, à chaque période de mesure par interpolation linéaire entre les deux mesures du foin témoin. Dans ces conditions, il apparaît : - que la capacité d'ingestion des moutons utilisés entraîne une sous-estimation de l'ingestibilité des fourrages en décembre-janvier et une surestimation en maiseptembre ; - que la correction proposée est tout à fait justifiée, puisque la liaison de toutes les ingestibilités avec un critère de prévision classique, en l'occurrence la teneur en parois totales (NDF), présente un écart type résiduel qui passe de 9,9 à $5,2 \mathrm{~g}$ de $\mathrm{MS} \cdot \mathrm{kg}^{-1}$ de $\mathrm{PV}^{0.75}$ après correction ; - que la correction fait apparaître que les quantités de foin et d'ensilage bien préparés sont comparables à celles des fourrages verts initiaux. Finalement il est proposé d'utiliser cette méthode de correction chaque fois que des mesures d'ingestibilités faites sur moutons, à des saisons différentes, et/ou sur des lots d'animaux différents, doivent être regroupées. (@ Elsevier / Inra)

ingestibilité / fourrages / capacité d'ingestion / moutons / méthodologie

\section{INTRODUCTION}

La mesure de l'ingestibilité des fourrages est difficile à standardiser, compte tenu de l'existence de nombreux facteurs modifiant la capacité d'ingestion des animaux consommateurs [5]. Parmi ces facteurs on peut citer la race, l'âge, l'état physiologique des animaux, les conditions extérieures (température, humidité, longueur du jour).

Ainsi la longueur du jour est un facteur classique dont l'effet, dans nos conditions, a été mesuré par Michalet-Doreau et Gatel $[14,15]$, ce travail se situant dans un vaste ensemble d'études initiées par Gordon [13] et abordées encore récemment par Deswysen et al. [4].

La maîtrise complète des facteurs de variation de la capacité d'ingestion est probablement impossible. Le dispositif en carré latin permet de contrôler les effets du milieu, mais il n'est pas utilisable pour l'étude des fourrages verts dont l'ingestibilité varie en continu. C'est pourquoi nous avons mis en place une méthodologie consistant à introduire un fourrage standard dans le dispositif de mesure, en nous inspirant de travaux passés $[1,11]$.

La méthode proposée permet d'estimer, à tout moment, la capacité d'ingestion des animaux pour corriger leurs quantités ingérées et obtenir une ingestibilité théoriquement indépendante des conditions de mesure. Le principe de cette méthodologie est de distribuer un foin standard avant et après toute séquence de mesures. Ce système permet de conserver la souplesse recherchée jusqu'à présent pour ces mesures : mesure séquentielle de l'ingestibilité des fourrages verts en les fauchant chaque jour, mesure séquentielle de l'ingestibilité des ensilages en ouvrant un seul silo à la fois, changement des moutons après chaque séquence pour avoir des animaux reposés

Les premiers résultats ont été présentés dans une courte note [7]. Les résultats de deux années supplémentaires, rassemblés aux premiers, sont donc présentés, avec pour objectif de proposer une méthodologie rénovée de mesure de l'ingestibilité des fourrages par des moutons standards.

\section{MATÉRIEL ET MÉTHODES}

Les mesures récapitulées concernent 3 années, 1996 à 1998 , pour un total de 20 séquences de mesures relatives à 51 fourrages différents. 


\subsection{Description d'une séquence de mesures}

Une séquence de mesures concerne un lot de moutons. Ce lot, maintenu dans des cages à métabolisme, reçoit pendant $10 \mathrm{j}$ le foin standard. II reçoit ensuite les fourrages expérimentaux (fourrages verts au printemps, foins et ensilages en automne et en hiver), 2 à 5 au maximum, pour des périodes successives de 2 à 3 semaines pour un fourrage. À la fin de la séquence les animaux reçoivent de nouveau le foin standard pendant $10 \mathrm{j}$. Cette durée de $10 \mathrm{j}$ a été choisie car elle est suffisante pour que les animaux atteignent un niveau d'ingestion stable (obtenu en 3-4 j) et le maintiennent pendant plusieurs jours.

\subsection{Animaux}

Dans tous les cas des lots de 6 béliers castrés de race Texel ont été utilisés. Ces moutons étaient âgés de 1 an à 2 ans, donc renouvelés chaque année. Ils pesaient entre 50 et $60 \mathrm{~kg}$ de poids vif.

\subsection{Capacité d'ingestion}

La capacité d'ingestion de chaque lot, au début, puis à la fin de la séquence de mesures, est estimée par la quantité ingérée (en g de MS par $\mathrm{kg}$ de $\mathrm{PV}^{0,75}$ ) de foin standard (en prenant les cinq derniers $\mathrm{j}$ sur les $10 \mathrm{j}$ de distribution). Ensuite la capacité d'ingestion au moment des mesures d'ingestibilité (six derniers j sur 14 à 2 I au total) est estimée par intrapolation, en supposant que son évolution entre le début et la fin d'une séquence est linéaire. On dispose au total de 42 mesures réelles ( 20 séquences plus deux mesures intra-séquence).

\subsection{Foin standard}

Une importante quantité de foin de dactyle au premier cycle a été récoltée dans de bonnes conditions au printemps 1995. Des analyses régulières ont été effectuées tout au long des 3 années d'étude. Ce foin a eu, en moyenne, une teneur en cendres de $68 \mathrm{~g}$ par $\mathrm{kg}$ de MS, une teneur en MAT de $88 \mathrm{~g}$ par $\mathrm{kg}$ de MS et une teneur en NDF de $710 \mathrm{~g}$ par $\mathrm{kg}$ de MS. Ces teneurs ont peu varié et n'ont pas subi de dérive systématique pendant les 3 années d'étude. Enfin, la digestibilité de la Matière Organique de ce foin, mesurée une fois, a été égale à $52 \%$.

\subsection{Fourrages expérimentaux}

Au total 51 fourrages ont été étudiés : 16 fourrages verts, 19 ensilages d'herbe, 14 foins et deux pailles. Parmi ces fourrages 44 ont un intérêt particulier : ils provenaient de 5 parcelles différentes (raygrass hybride, raygrass anglais, prairie naturelle, dactyle et luzerne) et ont été utilisés pour mesurer l'influence de 3 facteurs : le stade pour les fourrages verts, la fenaison et l'ensilage. Chaque espèce a été étudiée en vert, au cours du premier cycle de végétation (trois stades). Les ensilages et les foins ont tous été réalisés à partir des fourrages verts étudiés. La teneur en NDF de ces fourrages a été utilisée, comme critère de prévision de l'ingestibilité, pour valider la méthodologie de mesure utilisée. Des mesures ont également été réalisées sur deux pailles, un foin, un fourrage vert et trois ensilages. L'ensemble de ces fourrages était étudié dans le cadre d'un programme sur l'évaluation de la valeur azotée des fourrages [8].

\subsection{Analyses et mesures sur les fourrages}

Les ingestibilités, c'est-à-dire les quantités de MS volontairement ingérées chaque jour, calculées par différence entre quantités offertes et refusées, ont été mesurées sur les moutons en fixant les refus à 10-12\%. Les teneurs en MS des fourrages ont été déterminées à l'étuve, après séchage pendant 48 heures à $80^{\circ} \mathrm{C}$. Enfin, sur ces fourrages, après séchage à $60^{\circ} \mathrm{C}$ et broyage, la teneur en NDF de l'offert a été évalué selon la méthode de Goering et Van Soest [12].

\subsection{Calcul de l'ingestibilité corrigée}

La quantité moyenne de foin standard ingérée pendant les 3 années (42 mesures) a été égale à $50,0 \mathrm{~g}(+/-7,3)$ de MS par kg de PV ${ }^{0,75}$. L'ingestibilité des fourrages expérimentaux a donc été corrigée par rapport à cette valeur :

Ingestibilité corrigée $=$ Ingestibilité mesurée $\times$ 50 / capacité d'ingestion estimée lors de la mesure

\section{RÉSULTATS}

À l'intérieur d'un même lot de moutons la capacité d'ingestion des animaux pré- 
sente un écart type moyen de $5,5 \mathrm{~g}$ de $\mathrm{MS} \cdot \mathrm{kg}^{-1}$ de $\mathrm{PV}^{0,75}$, soit une variabilité moyenne de $10,3 \%$, pour 42 mesures sur le foin standard.

L'évolution de la capacité d'ingestion (mesurée par l'ingestion de foin standard) des différents lots de moutons au cours de l'année est donnée dans la figure 1. Cette capacité d'ingestion est la plus faible en décembre-janvier, avec une quantité de foin standard ingérée égale à $46 \mathrm{~g}$ de $\mathrm{MS} \cdot \mathrm{kg}^{-1}$ de $\mathrm{PV}^{0,75}$. Elle est de 49 fin février, puis élevée et relativement stable de fin avril à fin septembre (58 g de MS $\cdot \mathrm{kg}^{-1}$ de PV ${ }^{0.75}$ ).

Aux mêmes dates, la capacité d'ingestion est également variable entre les lots, avec un écart type inter-lots de 5,0 $\mathrm{g}$ de $\mathrm{MS} \cdot \mathrm{kg}^{-1}$ de PV0,75, en moyenne pour 7 dates.

L'effet de la correction des ingestibilités des 51 fourrages expérimentaux étudiés a été examiné à deux niveaux : sur la relation avec un critère de prévision, en l'occurrence la teneur en NDF (en g par kg de MS), et sur la comparaison des ingestibilités entre les fourrages verts initiaux et les fourrages conservés correspondants.
Ainsi, avant correction, on obtient pour les 51 fourrages (figure 2) :

$$
\begin{gathered}
\mathrm{INC}=-0,0743 \mathrm{NDF}+105,8 \\
\mathrm{R}^{2}=0,34 \text { et } \mathrm{ETR}=9,9
\end{gathered}
$$

Après correction, on obtient (figure 3) :

$$
\begin{gathered}
\mathrm{IC}=-0,0938 \mathrm{NDF}+114,5 \\
\mathrm{R}^{2}=0,75 \text { et } \mathrm{ETR}=5,2
\end{gathered}
$$

INC est l'ingestibilité non corrigée, IC l'ingestibilité corrigée et ETR l'écart type résiduel des régressions. La correction amène donc un gain de précision considérable.

La comparaison entre foins et fourrages verts initiaux porte sur 13 couples : l'ingestibilité de ces foins est égale à $85 \%$ de celles des fourrages verts avant correction et $98 \%$ après (tableau 1 ). Pour les ensilages la comparaison concerne 16 couples ( 8 sans et 8 avec acide formique comme conservateur) avec une ingestibilité égale à $91 \%$ de celle du fourrage vert avant correction et à $106 \%$ après, en cohérence d'ailleurs avec une baisse de $10 \%$ de la teneur en NDF. On note, dans le tableau $I$, une correction plus élevée pour les fourrages verts que les four-

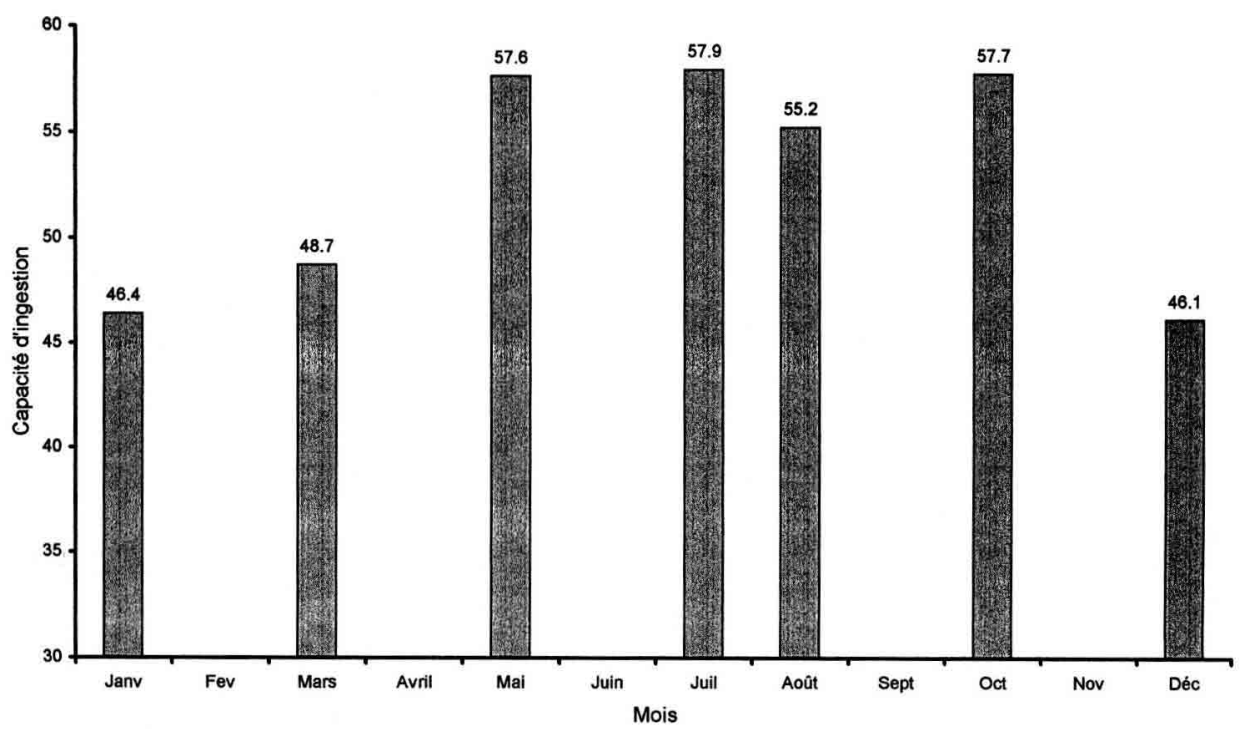

Figure 1. Évolution de la capacité d'ingestion des moutons au cours de l'année, exprimée en quantités de MS ingérées de foin standard $\left(\mathrm{g} \mathrm{de} \mathrm{MS} \cdot \mathrm{kg}^{-1} \mathrm{PV}{ }^{0.75}\right)$. 


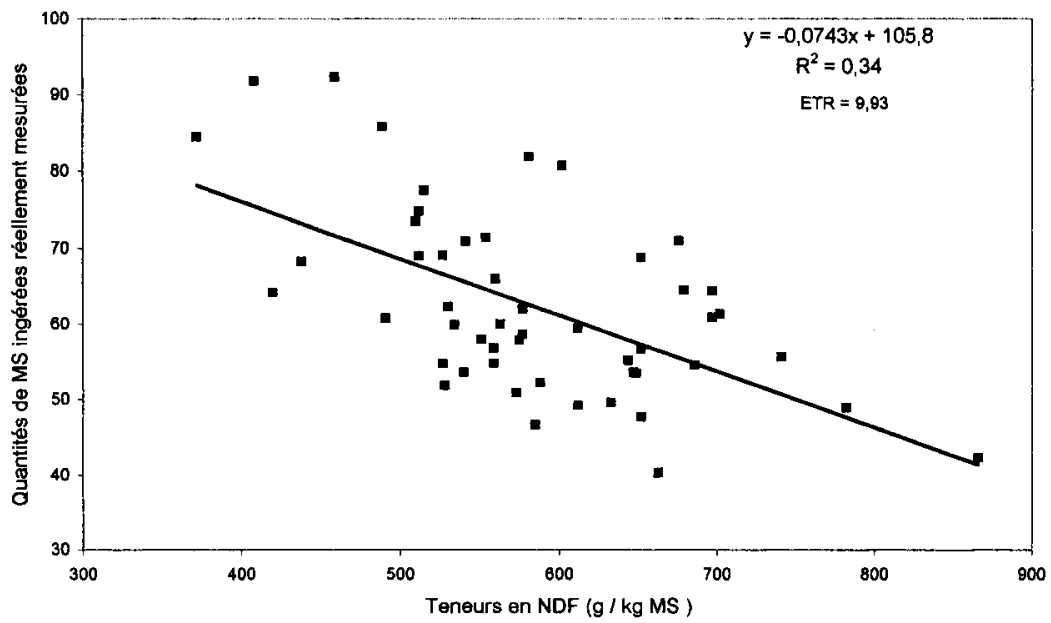

Figure 2. Relation entre quantités de MS ingérées réellement mesurées, non corrigées ( $\mathrm{g}$ de MS $\mathrm{kg}^{-1}$ $P V^{0.75}$ ), et teneurs en NDF des fourrages expérimentaux.

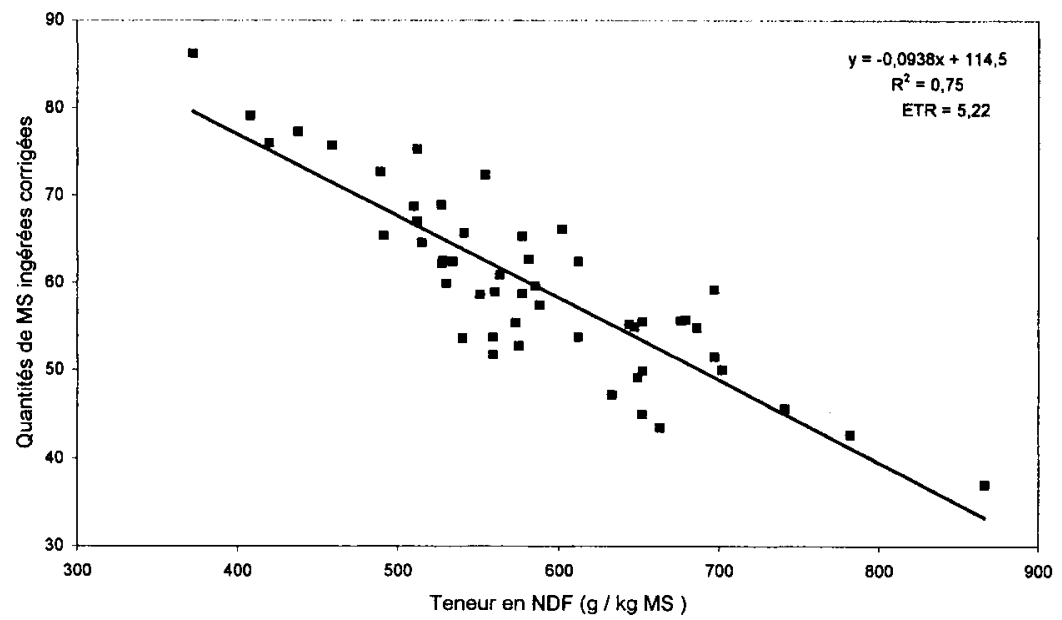

Figure 3. Relation entre quantités de MS ingérées corrigées par l'ingestion du fourrage témoin ( $\mathrm{g}$ de MS$\cdot \mathrm{kg}^{-1} \mathrm{PV}{ }^{0.75}$ ) et teneurs en NDF des fourrages expérimentaux.

rages conservés. Cela s'explique par le fait que les fourrages verts sont étudiés uniquement en période de capacité d'ingestion élevée, alors que les fourrages conservés le sont à la fois en période de capacité d'ingestion faible (novembre à février), mais aussi en période de capacité d'ingestion moyenne (octobre et mars) à élevée (septembre et avril).

\section{DISCUSSION}

L'évolution observée dans cette étude, au cours de l'année, de la capacité d'ingestion du mouton standard confirme les résultats de Michalet-Doreau et Gatel [14, 15], ainsi que ceux de Deswysen et al. [4]. Par rapport à la moyenne des résultats, l'écart entre les capacités d'ingestion minimale et 
Tableau I. Valeurs moyennes des ingestibilités et des teneurs en NDF des 44 fourrages inclus dans des comparaisons (stade, effet fenaison, effet ensilage).

\begin{tabular}{|c|c|c|c|c|}
\hline & \multirow{2}{*}{$\begin{array}{c}\text { Nombre } \\
\text { de fourrages } \\
\text { comparés }\end{array}$} & \multicolumn{2}{|c|}{ Ingestibilités (g MS $\cdot \mathrm{kg}^{-1} \mathrm{PV}{ }^{0.75}$ ) } & \multirow{2}{*}{$\begin{array}{c}\text { Teneurs en NDF } \\
\mathrm{g} \cdot \mathrm{kg}^{-1} \mathrm{MS}\end{array}$} \\
\hline & & mesurées & corrigées & \\
\hline \multicolumn{5}{|l|}{ Fourrages verts } \\
\hline Stade précoce & 5 & 76,8 & 68,1 & 519 \\
\hline Stade moyen & 5 & 67,7 & 58.8 & 585 \\
\hline Stade tardif & 5 & 60,3 & 51,5 & 629 \\
\hline Fourrages verts & 13 & 67,5 & 58,5 & 598 \\
\hline Foins & 13 & 57,3 & 57,3 & 622 \\
\hline Fourrages verts & 8 & 72,0 & 63,8 & 578 \\
\hline $\begin{array}{l}\text { Ensilages directs } \\
\quad \text { (sans conservateur) }\end{array}$ & 8 & 66,7 & 66,9 & 512 \\
\hline $\begin{array}{l}\text { Ensilages directs } \\
\quad \text { (avec acide formique) }\end{array}$ & 8 & 64,9 & 68,7 & 518 \\
\hline
\end{tabular}

maximale a été de $25 \%$. Cet écart était de $22 \%$ (mais sans mesure entre avril et septembre) pour Deswysen et al. [4], de $28 \%$ pour Michalet-Doreau et Gatel [14] et de $25 \%$ pour Michalet-Doreau et Gatel [15]. Cette capacité d'ingestion reste relativement stable de mai à septembre, comme l'avaient déjà noté Michalet-Doreau et Gatel [14], mais elle fluctue au cours de l'année. Les valeurs les plus faibles ont été trouvées en décembre-janvier, ce qui correspond bien aux observations de Michalet-Doreau et Gatel $[14,15]$ et de Deswysen et al. [4].

Cette variation annuelle de la capacité d'ingestion pose des problèmes pour mesurer l'ingestibilité des fourrages distribués à des saisons différentes. Ainsi, chez le mouton, les modifications d'ingestibilité supposées entraînées par la fenaison [3], soit $-19 \%$ pour des foins au sol par beau temps et $-24 \%$ pour des foins au sol avec un temps de séjour inférieur à $10 \mathrm{j}$, seraient dues avant tout à un effet de la saison. Il en est de même pour celles entraînées par l'ensilage [6], soit -16 à $-17 \%$ pour des ensilages directs avec conservateur. Cet effet ne semble pas exister chez les bovins [9], ce qui explique, pour ces derniers, des quantités ingérées de foin ou d'ensilage, préparés dans de bonnes conditions, très proches de celles des fourrages verts de départ.
La variabilité intra-lot a été comparable à celle déjà trouvée par Demarquilly et Chenost [2], +/- 6,9 g de MS par kg de PV ${ }^{0,75}$, soit une variabilité de $9 \%$, contre $10,3 \%$ dans nos mesures.

La variabilité inter lots a également été élevée. Il est donc nécessaire, pour mesurer l'ingestibilité des fourrages, de mettre en oeuvre une procédure de correction en ayant recours, soit :

- à des carrés latins,

- à une mesure sur un nombre élevé d'animaux,

- à une mesure préalable de la capacité d'ingestion [1]

- à une estimation continue de cette capacité d'ingestion (notre étude).

La correction des ingestibilités a fait diminuer de 9,9 à 5,2 l'écart type résiduel de la relation avec la teneur en NDF, ce qui correspond à une amélioration considérable. Dulphy et al. [10] avaient trouvé, pour des foins, la relation suivante :

$$
\mathrm{INC}=-0,099 \mathrm{NDF}+125,5
$$

$\mathrm{ETR}=8,3$ pour 115 foins de luzerne et de graminées,

avec $\mathrm{ETR}=7,0$ pour les seuls foins de graminées

$\mathrm{ETR}=7,2$ pour ceux de prairie naturelle et $\mathrm{ETR}=13,8$ pour ceux de luzerne. 
Mais il n'avait pas été possible de tenir compte de la variabilité inter-lots, ce qui explique probablement les écarts types résiduels élevés.

Les effets saison et lot expliquent aussi certainement les écarts types résiduels élevés des équations obtenues par Demarquilly et al. [3], équations reliant l'ingestibilité des fourrages à leur teneur en matières azotées totales et en cellulose brute, ainsi que la nécessité de séparer les types de fourrages pour obtenir une précision correcte. Toutefois ces résultats, tels qu'ils ont été présentés, ne sont que partiellement affectés par un effet de la saison. En effet les fourrages verts ont toujours été étudiés au printemps, voire en été, saison où la capacité d'ingestion des moutons est stable. Par contre les fourrages conservés ont toujours été étudiés à l'automne et en hiver. Il se pose bien alors le problème des variations de la capacité d'ingestion au cours de cette période. Dans les deux cas cependant, le nombre élevé de mesures et de lots utilisés a permis probablement d'obtenir des équations de prévision utilisables, bien que les écarts types résiduels soient élevés. La comparaison entre fourrages verts et fourrages conservés est, par contre, impossible si on ne corrige pas les variations de capacité d'ingestion des moutons.

Enfin, pour l'avenir, un point important reste le choix du fourrage standard. En effet l'utilisation d'un tel fourrage nécessite de bien le choisir. Pour des raisons pratiques l'utilisation d'un fourrage sec s'impose. Nous avons fait, pour l'instant, le choix d'utiliser un fourrage d'ingestibilité moyenne pour minimiser l'influence éventuelle de l'ingestion de ce foin sur celle des fourrages expérimentaux.

\section{CONCLUSION}

En définitive la mesure de l'ingestibilité des fourrages nécessite la mise en place de protocoles adaptés prenant en compte les variations de la capacité d'ingestion des moutons standards utilisés en France. Moyennant cela les méthodes de prévision de ces ingestibilités devraient s'améliorer nettement. La procédure que nous avons testée est évidemment lourde, mais elle semble nécessaire et, en tout cas, très utile pour améliorer les mesures de référence concernant l'ingestibilité des fourrages.

\section{RÉFÉRENCES}

[1] Abrams S.M., Harpster H.W., Wangsness P.J., Shenk J.S., Keck E., Rosenberger J.L., Use of a standard forage to reduce effects of animal variation on estimates of mean voluntary intake, J. Dairy Sci. 70 (1987) 1235-1240.

[2] Demarquilly C., Chenost M., Measurement of herbage intake by housed animals. in Leaver J.B. (éd.), Herbage intake handbook. A British Grassland Society publication (1982) pp. 95-112.

[3] Demarquilly C., Andrieu J., Weiss P., L'ingestibilité des fourrages verts et des foins et sa prévision ; in : Prévision de la valeur nutritive des aliments des ruminants, Inra Publications, Paris (1981) pp. 155-167.

[4] Deswysen A.G., Desprès L., Beckers F., Fisher V., Amouche E.H., Effect of daylength on voluntary intake, extent of digestion and chewing behaviour in housed sheep. Ann. Zootech. 44, suppl. 1 (1995) p. 259.

[5] Dulphy J.P., Demarquilly C., The regulation and prediction of feed intake in ruminants in relation to feed characteristics. Livest. Prod. Sci. 39 (1994) 1-12.

[6] Dulphy J.P., Michalet-Doreau B., Prévision de l'ingestibilité des ensilages d'herbe, in : Prévision de la valeur nutritive des aliments des ruminants, Inra Publications, Paris (1981) pp. 169-187.

[7] Dulphy J.P., Demarquilly C., Baumont R. L'Hotelier L., Effet de la saison sur la capacité d'ingestion du mouton standard : conséquences pour la mesure de l'ingestibilité, Renc. Rech. Rum. 4 (1997) p. 94

[8] Dulphy J.P., Demarquilly C., Baumont R. Jailler M., L’Hotelier L., Dragomir C., Study of modes of preparation of fresh and conserved forage samples for measurement of their dry matter and nitrogen degradations in the rumen. Ann. Zootech. (1999) 275-288.

[9] Dulphy J.P., Faverdin P., Micol D., Bocquier F., Révision du système des Unités d'encombrement (UE), Bull. Tech. CRZV Theix, Inra. 70 (1987) 35-48. 
[10] Dulphy J.P., Jailler M., Jamot J., Bousquet H., Amélioration de la prévision de la valeur alimentaire de certains foins au laboratoire. Fourrages 121 (1990) 65-78.

[11] Evans E.M., Potter J.F., The reproducibility of in vivo estimates of digestibility and voluntary digestible organic matter intake of grass varieties by sheep. Grass and Forage Sci. 39 (1984) 101-106.

[12] Goering H.K., van Soest P.J., Forage and fibre analyses, US Dept of Agriculture, Agric. Handbook No. 379 (1970), 20 p.
[13] Gordon J.G., Effect of time of the year on the roughage intake of housed sheep, Nature 204 (1964) 798-799.

[14] Michalet-Doreau B., Gatel F., Évolution au cours d'une année des quantités de foin ingérées par des béliers castrés, Ann. Zootech. 32 (1983) $459-464$

[15] Michalet-Doreau B., Gatel F, Évolution au cours d'une année des quantités de foin ingérées par des béliers castrés, Ann. Zootech. 37 (1988) $151-158$ 